\title{
Zoneamento ambiental dos banhados da Estação Ecológica do Taim, $\mathbf{R S}^{1}$
}

\author{
Environmental zoning of swamps in the Ecological Station of \\ Taim, Rs, Brazil
}

\author{
Fabio Charão Kurtz ${ }^{2}$ José Sales Mariano da Rocha ${ }^{3}$ Silvia Margareti de Juli \\ Morais Kurtz ${ }^{4}$ Adroaldo Dias Robaina ${ }^{5}$ Sandra Maria Garcia ${ }^{6}$ \\ Alessandro Herbert de Oliveira Santos ${ }^{6}$ Paulo Roberto Jaques Dill $^{7}$ \\ Paulo Roberto Vasques de Ataides $^{8}$ Fabrina Bolzan ${ }^{9}$
}

\section{RESUMO}

O "Zoneamento Ambiental dos Banhados da Estação Ecológica do Taim” permitiu avaliar a deterioração ambiental dos ecossistemas existentes na Estação Ecológica do Taim (ESEC/TAIM), municípios de Rio Grande e Santa Vitória do Palmar (RS). Consideraram-se dois tipos distintos de ecossistemas: $o$ do Banhado (ECO1 = Ecossistema Límnico) e o da Planície Marítima (ECO2 = Ecossistema Planície Maritima). A ECO (ECO1 + ECO2) apresentou $64 \%$ de classe APP (Área dé Preservação Permanente), 27,6\% de ACP (Área de Conservação Permanente), 5,6\% de AUO (Uso e Ocupação); em menor porcentagem encontrou-se a classe AR (Área de Restauração) com 2,8\%. A deterioração ambiental média para o ECO1 foi de 10,32\%, sendo menor que a ECO2 $(23,94 \%)$. A deterioração ambiental da ESEC/TAIM (ECO) ficou em 13,65\%. Com relação à análise de regressão para ECO1, concluiu-se que com 06 parâmetros ambientais se obteve a precisão de 95\%, enquanto que, com 04 parâmetros ambientais obteve-se a mesma precisão para a ECO2. Com relação à análise fatorial concluiu-se que essa técnica permitiu conhecer a estrutura dos dados, mostrando as correlações entre cada variável (classes de exuberância) e seu respectivo fator, entretanto não foi possivel separar grupos ou quantificar a influência de uma ou mais variáveis sobre outra de interesse (variável resposta), como por exemplo: quantos parâmetros são necessários para elaborar um zoneamento ambiental, sendo esta pergunta respondida pela análise de regressão. Recomenda-se que o zoneamento ambiental seja elaborado pelos órgãos públicos ambientais nas demais estações ecológicas e nas unidades de conservação.

Palavras-chave: zoneamento ambiental, banhado, estação ecológica.

\section{ABSTRACT}

The "Environmental Zoning of swamps in the Ecological Station of Taim (RS)" allowed to evaluate an environmental deterioration of the existent ecosystems in the Ecological Station of Taim (ESEC/TAIM); municipal districts of Rio Grande and Santa Vitória do Palmar (RS). Two types of different ecosystems were considered: swamps zone (ECO1 = Ecossistema Limnico) and Marine Plain (ECO2 = Ecossistema Planicie Maritima). The total ecosystem (ECO1 + ECO2) presented $64 \%$ of the class APP (Permanent Preservation Area),

${ }^{1}$ Dissertação de Mestrado em Engenharia Agrícola do PPGEA / DER / CCR / UFSM - RS, do primeiro autor, realizado com apoio da CAPES e da Sociedade Brasileira de Cultura Japonesa de São Paulo.

${ }^{2}$ Engenheiro Florestal, MSc. Doutorando do PPGEA, Departamento de Engenharia Rural (DER), Centro de Ciências Rurais (CCR), Universidade Federal de Santa Maria (UFSM) 97105-900, Santa Maria, RS. E-mail: fckurtz@mail.ufsm.br. Autor para correspondência.

${ }^{3}$ Orientador, Engenheiro Florestal, Dr., Livre Docente, Professor Titular, Chefe do DER, CCR, UFSM.

${ }^{4}$ Engenheira Florestal, Professora, Doutora, DER, CCR, UFSM.

${ }^{5}$ Engenheiro Agrônomo, Dr., Professor, DER, CCR, UFSM.

${ }^{6}$ Engenheiros Florestais, MSc., Doutorandos do PPGEA, DER, CCR, UFSM.

${ }^{7}$ Engenheiro Florestal, MSc do LAPAF, DER, CCR, UFSM.

${ }^{8}$ Engenheira Florestal, MSc., DEFAP - Departamento de Florestas e Áreas Protegidas da SEMA, RS.

${ }^{9}$ Acadêmica do Curso de Engenharia Florestal, Estagiária do LAPAF. 
27,6\% of ACP (Permanent Conservation Area), 5,6\% of AUO (Use and Occupation Area); in smaller percentage it was observed the class AR (Restoration Area) with 2,8\%. The deterioration environmental average for the ECO1 was $10.32 \%$, smaller that ECO2 $(23.94 \%)$. The environmental deterioration of ESEC/TAIM (ECOTOTAL) was 13,65\%. Regarding the regression analysis for ECO1, it was obtained a precision of $95 \%$, with 06 environmental parameters whereas ECO2 with 04 environmental parameters obtained the same precision. With the factorial analysis it was possible to know the structure of the data, showing the correlations between each variable (exuberance classes) and its respective factor. However, it was not possible to separate groups or quantify the influence of one or more variables over another (answer variable), as for example: how many parameters are necessary to elaborate an environmental zoning; this question was answered by the regression analysis. It is recommended that the environmental zoning be elaborated by the public organizations in other ecological stations and conservation units.

Key words: environmental zoning, swamp regions, ecological station.

\section{INTRODUÇÃO}

Como estudo de um caso escolheu-se o Banhado ${ }^{a}$ do Taim, classificado como estação ecológica pelo IBAMA, localizado nos Municípios de Rio Grande e Santa Vitória do Palmar (RS). A área estudada é formada por dois ecossistemas que são de extrema importância na manutenção e preservação da fauna e flora locais em função do vasto número de espécies ainda existentes. A maioria dos conflitos ambientais naquele ecossistema ocorre devido à crescente presença do homem, trazendo consigo a construção de casas e estradas, o aumento das áreas agrícolas (principalmente arrozais) e inúmeras outras atividades que, se não forem corretamente introduzidas, poderão conduzir a sérios desequilíbrios ambientais, comprometendo até a própria existência do banhado. É do "habitat" que os rodeia que os animais retiram o alimento, a água e o abrigo, indispensáveis para a sua sobrevivência. Assim, torna-se necessária a aplicação de medidas que garantam a exploração racional dos recursos naturais disponíveis, caso se deseje assegurar a preservação e conservação da fauna e flora presentes naquela estação ecológica.

O zoneamento ambiental faz parte de um conjunto de projetos ambientais desenvolvidos no sentido de fornecer orientação para o desenvolvimento sustentável dos recursos naturais, especificamente solo, água, vegetação e fauna silvestre.

Para MILANO (1993), todas as informações relativas ao zoneamento das unidades de conservação devem estar reunidas em uma carta geral de zoneamento. O uso do zoneamento apresenta ainda as seguintes vantagens: a) Permite que se determine limites de irreversibilidade e pontos de fragilidade biológica antes que se tomem decisões sobre o uso de cada área, evitando-se danos irreversíveis, tendo, portanto, caráter preventivo; b) Permite a identificação de atividades adequadas para cada setor da unidade de conservação e seu respectivo manejo; c) Permite pela flexibilidade da metodologia, aumentar ou diminuir o número de parâmetros adaptando-os às realidades de cada banhado (cada região).

$\mathrm{O}$ presente trabalho visou à avaliação da deterioração ambiental atual da Estação Ecológica do Taim, sendo avaliados 08 parâmetros ambientais, para dois ecossistemas da referida estação: ecossistema límnico (ECO1) e planície marítima (ECO2). Os parâmetros avaliados foram os seguintes (Tabela 1): vegetação (V1), lacustricidade (L2), fauna silvestre (F3), paisagem nativa (presença de ação humana) (P4), ocupação humana (habitações) (O5), uso da terra (agricultura, pecuária, floresta) (U6), acesso à área (A7) e dunas móveis (D8).

Em vista do exposto, este trabalho teve como objetivo relatar a elaboração do "Zoneamento Ambiental no Banhado do Taim". Para isso, utilizaramse técnicas propostas por Rocha (1997), visando apresentar e discutir resultados em relação ao zoneamento ambiental, estudando-se 08 parâmetros por ecossistema, selecionando-se, com modelos matemáticos adequados $\mathrm{CZ}_{\mathrm{ECO} !}$ e $\mathrm{CZ}_{\mathrm{ECO} 2}$ ), quais os parâmetros mínimos e necessários para se elaborar o referido zoneamento.

\section{MATERIAL EMÉTODOS}

Localizada na Planície Costeira do Estado do Rio Grande do Sul, a Estação Ecológica do Taim (ESEC/TAIM) foi criada pelo Decreto $\mathrm{n}^{\circ} 92.963 \mathrm{em} 21$ de Julho de 1986 e possui uma área de 33.995,00 ha (Ibama, 1989).

A metodologia proposta e desenvolvida por Rocha (1997), aplicada na APA (Área de Proteção Ambiental) de Santa Rita e Reserva Ecológica do Saco da Pedra em Marechal Deodoro (AL), foi adaptada para o presente trabalho. Esta metodologia é semelhante à proposta pela Secretaria do Meio Ambiente do Estado de São Paulo. No presente trabalho, propôs-se a divisão da área em 04 classes distintas: Área de Preservação Permanente (APP), Área de Conservação Permanente (ACP), Área de Restauração (AR), Área de Uso e Ocupação (AUO).

A presente metodologia selecionou 08 parâmetros em unidades homogêneas, aplicando-os aos dois ecossistemas definidos, quando foi realizada a revisão de literatura (ECO1 - Ecossistema Límnico e ECO2 - Ecossistema Planície Marítima).

A figura 1 mostra uma sinopse da metodologia, onde se tem uma idéia geral do trabalho executado para que o objetivo proposto fosse alcançado. 


\section{RESULTADOS E DISCUSSÃO} inferir que:

Conforme os valores da tabela 1 , pode-se

Como conceito geral à deterioração ambiental varia de $0 \%$ (sem deterioração) a $100 \%$ (máxima deterioração), valores de y que figuram na equação da reta.

Para os valores mínimos $\left(\sum\right.$ Mín. $\left.=8\right)$ temse: $08 . \mathrm{a}+\mathrm{b}=0$

Quando $x=8=>y=0 \%$; então $=>\mathrm{a} \cdot x+\mathrm{b}=$

Para os valores máximos ( $\sum$ Máx. $\left.=42\right)$ temse:

Quando $x=42=>y=100 \%$; então $=>\mathrm{a} . x+\mathrm{b}$ $=042 \cdot \mathrm{a}+\mathrm{b}=100$, onde o sistema de equação fica:

$$
\begin{aligned}
& 8 . a+b=0(-1) \\
& 42 . a+b=100
\end{aligned}
$$

Sendo: $\mathrm{a}=2,9412 ; \mathrm{b}=-23,5294$, então $\mathrm{y}=$ 2,9412.x-23,5294.

Com esta equação, calculou-se o valor de $y$ em função de cada indicador total $x$ (por hexágono). Com este valor calculado de $y$, definiram-se as classes ambientais. $\mathrm{O}$ valor de $y$ significa unidade crítica de deterioração e $x$ o valor do indicador total encontrado.

\section{Zoneamento ambiental}

Para elaboração final da carta de zoneamento ambiental foram usados hexágonos devido a seus cantos serem arredondados, já que na natureza não há limites quebrados/geométricos. A cada classe correspondeu uma cor anteriormente estipulada por ROCHA (1997): APP (verde = 1), ACP $($ cinza = 2), AR $($ amarelo $=3)$ e AUO (vermelho $=4)$.

A figura 2 representa a "Carta de Zoneamento Ambiental em Banhados para ESEC/ TAIM".

Com base na figura 2, verificou-se que para ECO existem aproximadamente $64 \%$ da classe APP, ocupandó a maior área da ESEC/TAIM; 27,6\% de ACP; $5,6 \%$ de AUO, e em menor porcentagem a classe AR com $2,8 \%$. As deteriorações ambientais médias foram: ECO1 10,32\%, ECO2 23,94\% e ECO 13,65\%.

\section{Análise estatística aplicada ao zoneamento ambiental}

\section{Análise de regressão}

Os resultados obtidos por meio da análise dos pesos atribuídos em cada parâmetro ambiental/ hexágono foram utilizados na análise de regressão. Para tanto, utilizou-se o método "Stepwise/forward", o qual consiste em fazer a regressão passo a passo, tendo

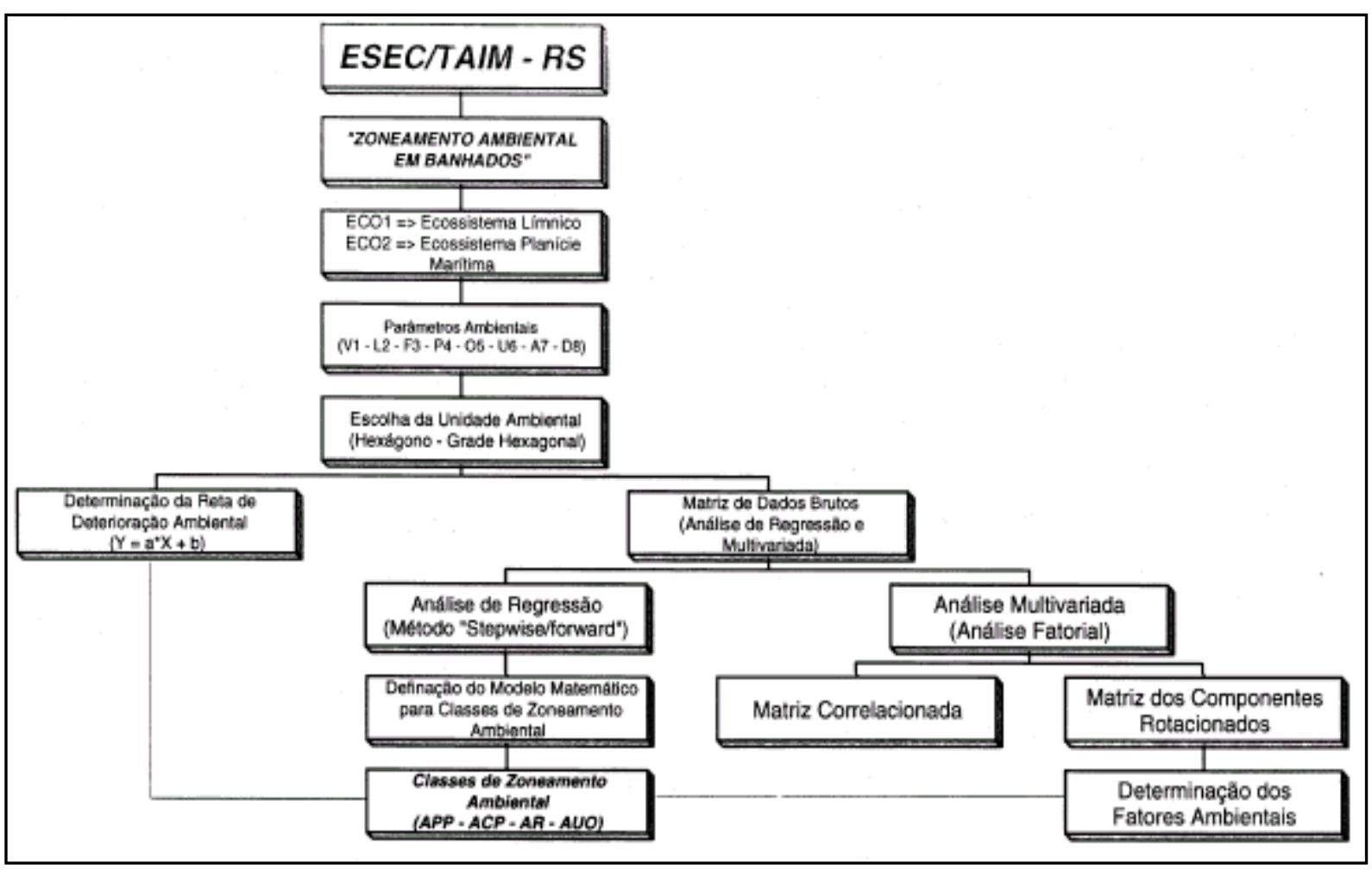

Figura 1 - Sinopse da Metodologia aplicada no "Zoneamento Ambiental em Banhados”da ESEC/TAIM - RS.

Ciência Rural, v. 33, n. 1, jan-fev, 2003. 


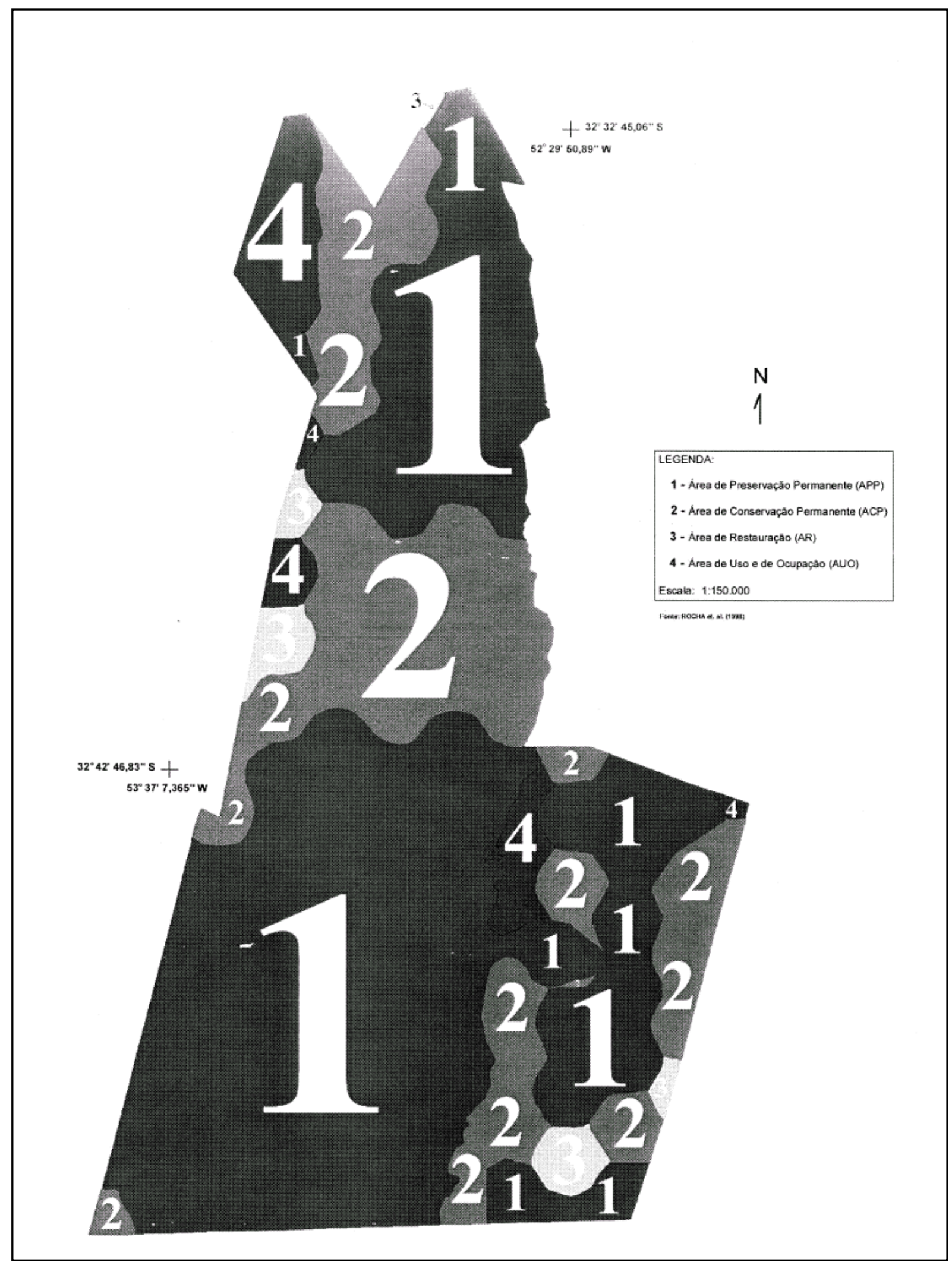

Figura 2 - Carta de Zoneamento Ambiental em Banhado (ESEC/TAIM - RS).

Ciência Rural, v. 33, n. 1, jan-fev, 2003. 
como objetivo analisar a precisão e o melhor modelo matemático, ao introduzir na equação de regressão as variáveis independentes uma a uma, em relação à quantidade mínima de parâmetros ambientais necessários para elaborar o zoneamento ambiental. Neste procedimento de regressão, foram consideradas como variáveis independentes os 08 parâmetros ambientais representados na tabela 1 , e como variável dependente a Classe de Zoneamento Ambiental (CZ) com 04 classes distintas representadas na divisão do zoneamento ambiental: APP - peso ambiental $=1 ; \mathrm{ACP}$ $=2 ; \mathrm{AR}=3 ; \mathrm{AUO}=4$.

Definidas as variáveis independentes e dependentes, processou-se a análise de regressão, utilizando-se o software estatístico SAS - Statistical Analysis System, versão 6.08 para MS-DOS (SAS, 1993), para ECO1 e ECO2.

Através desta análise, chegou-se aos seguintes resultados para ECO1: no passo 6 (step 6) foi adicionada a variável $\mathrm{O} 5$, com um valor $\mathrm{F}$ de 4,50, $(\mathrm{p}=0,0364)$, porém com $\mathrm{C}(\mathrm{p})=9,589$, o que leva a ser escolhido este modelo para definir a variável $\mathrm{CZ}$, pois, este valor significa que está mais próximo da soma da quantidade de variáveis (dependente e independentes) que possui o zoneamento ambiental. O parâmetro ambiental $L 2$ não entrou em a análise de regressão, pois não atingiu o nível de significância de 0,5 para que pudesse entrar no modelo. Uma observação importante é que este parâmetro pouco contribuiu estatisticamente para a análise, pois a grande maioria dos pesos dada para os hexágonos foi de valor 1, sendo a melhor situação ambiental no presente caso.

O parâmetro ambiental $V 1$ foi excluído, porque estava abaixo do valor do número de variáveis existentes em a análise, que era 9 , e sua contribuição foi de somente de $0,002 \%$ em relação ao parâmetro ambiental anterior, não justificando sua inclusão no modelo.

Tabela 1 - Parâmetros e valores ambientais utilizados no Zoneamento Ambiental em Banhados.

\begin{tabular}{llll}
\hline \multirow{2}{*}{ CÓDIGO } & \multirow{2}{*}{$\begin{array}{c}\text { PARÂMETRO } \\
\text { AMBIENTAL }\end{array}$} & \multicolumn{2}{c}{ VALOR AMBIENTAL } \\
& EONDERADO \\
& Eegetação & $1=>4$ & $1=>4$ \\
V1 & Lacustricidade & $1=>4$ & $1=>4$ \\
L2 & Fauna Silvestre & $1=>4$ & $1=>4$ \\
F3 & Paisagem Nativa & $1=>5$ & $1=>5$ \\
P4 & Ocupação Humana & $1=>4$ & $1=>4$ \\
O5 & Uso da Terra & $1=>5$ & $1=>5$ \\
U6 & Acesso à Área & $1=>6$ & $1=>6$ \\
A7 & Dunas Móveis & $1=>10$ & $1=>10$ \\
D8 & TOTAIS & $8=>42$ & $8=>42$ \\
& & &
\end{tabular}

Logo, observa-se que o modelo deve ser representado pelo obtido no passo 6 , sendo expresso por: $C Z=-1,059-0,359 \cdot D 8+0,254 \cdot P 4+0,379 \cdot F 3$ $+0,203 . A 7+0,283 \cdot U 6+0,332.05$.

Depois de escolhido o modelo para ECO1, realizou-se a análise de regressão para este modelo, chegando aos seguintes resultados: Coeficiente de Determinação igual a 0,95 e um Coeficiente de Variação de $13,16 \%$, juntamente com as estimativas para todas as observações (hexágonos) feitas no zoneamento ambiental. Pode-se se dizer que, com 06 parâmetros ambientais para este ecossistema, obteve-se um nível satisfatório de precisão de $95 \%$, aproximadamente.

Para ECO2, chegou-se aos seguintes resultados: no passo 4 (step 4) foi adicionada a variável $\mathrm{D} 8$, com um valor $\mathrm{F}$ de $25,82(\mathrm{p}=0,0001)$, porém com $\mathrm{C}(\mathrm{p})=5,0$, o que levou este modelo a ser escolhido para definir a variável $\mathrm{CZ}$, pois, este valor significa que está mais próximo da soma da quantidade de variáveis (dependente e independentes) que possui o zoneamento ambiental. Os parâmetros $F 3, O 5, U 6$ e $A 7$, não foram escolhidos em a análise de regressão, pois o programa não reconheceu o valor 0,5 , nível de significância para que entrasse no modelo, justificando e exclusão desses 04 parâmetros ambientais.

Observa-se que o modelo deve ser representado pelo obtido no passo 4 , sendo expresso por: $C Z_{E C 02}=-0,927+0,278 \cdot V 1+0,325 \cdot L 2+0,813 \cdot P 4$ $+0,169 . D 8$.

Depois de escolhido o modelo para ECO2, realizou-se a análise de regressão para este modelo, chegando aos seguintes resultados: Coeficiente de Determinação igual a 0,95 e um Coeficiente de Variação de $12,94 \%$, juntamente com as estimativas para todas as observações (hexágonos) feitas no zoneamento ambiental. Pode-se dizer também que, com 04 parâmetros ambientais para este ecossistema, obtevese um nível satisfatório de precisão de 95\%, aproximadamente.

Com base nestas equações para classes de zoneamento ambiental de ECO1 e ECO2, qualquer trabalho envolvendo zoneamento ambiental em área de estudo similar poderá usar os parâmetros ambientais contidos nas fórmulas acima.

\section{Análise fatorial}

Utilizou-se também, com os resultados obtidos através da análise dos valores ambientais/ pesos atribuídos a cada parâmetro ambiental/ hexágono, a análise multivariada. Para tanto se utilizou a técnica da análise fatorial, tendo como objetivo averiguar a existência de um número reduzido de fatores, que expliquem o máximo das correlações observadas entre as variáveis estudadas. 
Definiram-se as variáveis (parâmetros ambientais) e processou-se a análise de fatorial usando o método das "Componentes Principais", utilizandose o software estatístico SPSS - Statistical Package for Social Sciences, versão 7.5 for Windows (SSPS, 1996).

Como critério de seleção das variáveis, utilizou-se o método dos componentes principais, com autovalores maiores que um para ECO1 e ECO2. Dessa forma, obtiveram-se os componentes, sendo que 04 explicam 88,97\% da variância total para ECO1 e 02 explicam $92,68 \%$ para ECO2.

Com base na literatura (BATISTA, 1990), quando se usa a matriz de correlação para ECO1 e ECO2, pode-se afirmar que é recomendável que se tome tantos fatores quanto forem os autovalores maiores que 1,0. A partir dos valores encontrados na matriz dos componentes rotacionados na tabela 3 ou matriz dos parâmetros ambientais, onde são interpretados como coeficientes de correlação entre cada variável e as componentes fatoriais, pode-se comprovar as vantagens da utilização desse método estatístico para o presente trabalho.

Através do resultado da análise fatorial pode-se dizer que, tomando os 04 fatores comuns reduziu-se a matriz de dados brutos da ordem de $109 \mathrm{x}$ 08, chegando numa matriz rotacionada da ordem de 109 x 04, mantendo-se um nível de informação de 88,97\% para ECO1. Para ECO2, 02 fatores comuns reduziram a matriz de dados brutos da ordem de $35 \times 08$, chegando a uma matriz rotacionada da ordem de $35 \times 02$, mantendose um nível de informação de $92,68 \%$.

Verifica-se também que, através da análise fatorial, foram extraídos 04 fatores para ECO1 e 02 fatores para ECO2, os quais por definição são independentes, isto é, não correlacionados entre si e os valores são interpretados como coeficientes de correlação entre cada variável e o fator. Na interpretação dos resultados obtidos da matriz dos componentes rotacionados, onde se resume na denominação dos fatores identificados pela análise fatorial com as variáveis em cada fator para ECO1 e ECO2.

É importante ressaltar que, além dos 04 fatores construídos não serem correlacionados, e também como não foi possível observar cada fator comum, torna-se adequado fazer a caracterização de cada variável, ou seja, com base na tabela 4 , observase que para ECO1:

Fator 1: para Dunas Móveis (D8) e Lacustricidade (L2), mostraram correlações de 0,971 e 0,954, respectivamente. São os variáveis com maior pesos da combinação linear que definem esse fator, caracterizando o fator resultante Ecossistema Taim " $A$ ";
Fator 2: esse fator foi representado pelo Uso da Terra (U6), Acesso à Área (A7) e Paisagem Nativa (P4) com correlações positivas de 0,908, 0,860 e 0,797, respectivamente, caracterizando o fator resultante como Geográfico;

Fator 3: o fator apresenta as variáveis Fauna Silvestre (F3) e Vegetação (V1), com 0,956 e 0,758, respectivamente, caracterizando o fator resultante das variáveis como Fauna \& Flora;

Fator 4: a variável que mais evidencia esse fator é a Ocupação Humana (O5) como mostra sua correlação positiva de 0,985 , caracterizando o fator resultante da variável como Ocupação Humana.

Também se ressalta para ECO2 que, além dos 02 fatores construídos não serem correlacionados, e também como não foi possível observar cada fator comum, torna-se adequado fazer a caracterização de cada variável, ou seja, com base na tabela 4 , observase que:

Fator 1: esse fator foi representado pela Ocupação Humana (O5), Paisagem Nativa (P4) e Uso da Terra (U6) com alta correlação positiva de 0,998 para os três, caracterizando o fator resultante como Geográfico;

Fator 2: o fator apresenta as variáveis Vegetação (V1), Dunas Móveis e Lacustricidade (L2) com $0,942,0,921$ e 0,883 , respectivamente, caracterizando o fator resultante das variáveis como Ecossistema Taim " $B$ ”;

Ressalva-se que o presente zoneamento ambiental foi realizado numa situação de outono/ inverno, quando o nível das águas se encontrava mais elevado. Por coincidência, a imagem de satélite obtida no inverno de 1996, foi imprescindível na avaliação de parâmetros (caso das dunas móveis e lacustricidade). Desta forma, a classificação das classes de zoneamento ambiental deve ser considerada para o inverno, devendo estes resultados, numa situação de verão, serem reavaliados.

\section{CONCLUSÕES}

Em função dos objetivos propostos no desenvolvimento da metodologia de Zoneamento Ambiental, elaborado a partir de parâmetros ambientais/ variáveis ambientais e, em função dos resultados obtidos, conclui-se que: a) A metodologia utilizada para o "Zoneamento Ambiental em Banhados" na Estação Ecológica do Taim (estudo de caso) levou a resultados convincentes com relação à realidade da área de estudo; b) $\mathrm{Na}$ Classe de Zoneamento Ambiental, Área de Preservação Permanente (APP), onde se incluem grandes partes do banhado e das lagoas, deverá ser 
proibida a entrada de pessoas em virtude da fragilidade do ecossistema, exceção aos trabalhos de pesquisas; c) $\mathrm{Na}$ Classe de Zoneamento Ambiental, Área de Restauração (AR) as áreas deverão ser transformadas em Áreas de Conservação Permanente (ACP) ou Áreas de Preservação Permanente (APP), evitando-se aí, futuramente, ações antrópicas; d) ECO1, ECO2 e $\mathrm{ECO}_{\mathrm{TOTAL}}$ as deteriorações nas classes de zoneamento ambiental aumentam na mesma proporção que o valor ambiental para cada classe; e) Os melhores modelos com relação à análise de regressão para ECO1 e ECO2, foram: para ECO1, o $6^{\circ}$ modelo matemático, composto por 06 parâmetros ambientais, assim constituídos: Dunas Móveis, Paisagem Nativa, Fauna Silvestre, Acesso à Área, Uso da Terra e Ocupação Humana e para $\mathrm{ECO} 2 \mathrm{o} 4^{\circ}$ modelo matemático, composto por quatro parâmetros ambientais assim constituídos: Vegetação, Lacustricidade, Paisagem Nativa e Dunas Móveis; f) A análise fatorial mostrou para ECO1 08 variáveis ambientais e, para ECO2 06 variáveis ambientais para representarem as matrizes de dados brutos, sendo que estas forneceram parâmetros ambientais suficientes para a identificação de grupos homogêneos; g) A aplicação da presente metodologia pelos órgãos públicos ambientais responsáveis pelas demais unidades de conservação (estações ecológicas, entre outras), poderá ser uma ferramenta importante e útil para tomada de decisões.
FONTES DE AQUISIÇÃO

a - Alagadiço coberto de vegetação. Sinônimo "pântano".

\section{AGRADECIMENTOS}

Ao Programa de Pós-graduação em Engenharia Agrícola - PPGEA (CCR/UFSM - RS), pela participação no Mestrado em Engenharia Agrícola. A CAPES pela concessão da bolsa de estudo para a realização deste curso. À Sociedade Brasileira de Cultura Japonesa e ao Banco Sumitomo de São Paulo por reconhecer nacionalmente e apoiar a dissertação de mestrado com o Prêmio Auxílio à Pesquisa 98.

\section{REFERÊNCIAS BIBLIOGRÁFICAS}

BATISTA, J. L. M. Análise multivariada no planejamento de extensão florestal: subsídios para uma política de reocupação de áreas. 1990. 137f. Dissertação (Mestrado em Ciências Florestais) - Universidade Federal do Paraná, 1990.

IBAMA. Unidades de conservação do Brasil: Parques nacionais e reservas biológicas. Brasília, 1989. v. 1, 192p.

MILANO, M. S. Unidades de conservação: conceitos básicos e princípios gerais de planejamento, manejo administração. Curitiba, 1993. 63p.

ROCHA, J. S. M. Manual de projetos ambientais. Santa Maria : UFSM, 1997. 446p.

SAS - Statistical Analysis System, versão 6.08 para MSDOS. Carry, 1993.

SPSS - STATISTICAL PACKAGE FOR THE SOCIAL SCIENCES Programa de computador, ambiente Windows versão 7.5 for Windows (SSPS, 1996). 